\title{
Entwicklungsperspektiven von Produktionsarbeit
}

\author{
Hartmut Hirsch-Kreinsen
}

Die Einführung von Industrie 4.0-Systemen ist von großem arbeitssoziologischen wie auch arbeitspolitischem Interesse. Denn es liegt auf der Hand, und es ist in der Debatte um Industrie 4.0 unumstritten, dass smarte Produktionssysteme im Fall ihrer breiten Durchsetzung die bisherige Landschaft der Arbeit in der industriellen Produktion nachhaltig verändern werden (Geisberger und Broy 2012; Kurz 2013; Spath et al. 2013). Auszugehen ist von einem disruptiven Wandel von Prozess- und Arbeitsstrukturen vor allem dadurch, dass die bisherigen sequentiell und ex-ante optimierten Abläufen durch in Echtzeit gesteuerte Prozesse ersetzt werden. Im Folgenden wird nun eine erste Einschätzung möglicher Wandlungstendenzen und damit auch Gestaltungsherausforderungen von Produktionsarbeit im Kontext der Einführung von smarten Produktionssystemen vorgelegt. Der Fokus der folgenden Analyse richtet sich primär auf mögliche innerbetriebliche Wandlungstendenzen der Arbeit. Ausgeklammert werden Veränderungstendenzen überbetrieblicher Produktionsbeziehungen. Methodisch basiert die folgende Argumentation auf einer Durchsicht und einer systematischen Zusammenfassung der vorliegenden Literatur aus dem Bereich der sozialwissenschaftlich orientierten Arbeitsforschung, die sich mehr oder weniger explizit mit dem Wandel von Produktionsarbeit unter den Bedingungen fortgeschritten automatisierter Systeme befasst.

\section{Dimensionen des Wandels}

Die Analyse des Zusammenspiels der neuen Technologie und der dadurch induzierten personellen und organisatorischen Veränderungen erfordert grundsätzlich den Blick auf das Gesamtsystem der Produktion und die hier wirksamen Zusammenhänge. Die neuen

Der Originaltext dieses Beitrags wurde überarbeitet. Das vollständige Korrekturverzeichnis finden Sie am Ende des Buchs und online unter http://dx.doi.org/10.1007/978-3-662-45915-7_16.

H. Hirsch-Kreinsen $(\bowtie)$

Technische Universität Dortmund, Wirtschafts- und Sozialwissenschaftliche Fakultät, Wirtschaftsund Industriesoziologie, 44221 Dortmund, Deutschland

e-mail: Hartmut.Hirsch-Kreinsen@tu-dortmund.de

(C) The Author(s) 2015

A. Botthof, E.A. Hartmann (Hrsg.), Zukunft der Arbeit in Industrie 4.0,

DOI 10.1007/978-3-662-45915-7_10 
Produktionssysteme sind daher, einer lange zurückreichenden arbeitssoziologischen Debatte folgend (Trist und Bamforth 1951; zusammenfassend Sydow 1985), als soziotechnische Systeme zu verstehen. Denn allein dadurch sind hinreichend begründete Aussagen über die Entwicklungsperspektiven Gestaltungsmöglichkeiten für Arbeit möglich (Forschungsunion und acatech 2013). Daher muss auch von einem weiten Verständnis von Produktionsarbeit ausgegangen werden. Denn betroffen von den absehbaren Wandlungstendenzen sind alle direkt und indirekt wertschöpfenden Tätigkeiten in Industriebetrieben; das heißt, betroffen sind die operative Ebene des Fertigungspersonals, wie aber auch die Bereiche des unteren und mittleren Managements von Produktionsprozessen sowie die Gruppe der technischen Experten. Folgt man diesen kategorialen Bestimmungen, so erweisen sich Wandlungstendenzen und Gestaltungsmöglichkeiten von Produktionsarbeit in den folgenden Dimensionen als relevant.

\section{Mensch-Maschine-Schnittstelle}

Ausgangspunkt ist die Dimension der unmittelbaren Mensch-Maschine Interaktion. Als zentrale Herausforderung der Arbeitsgestaltung erweist sich hierbei das Problem, inwieweit die Beschäftigten unmittelbar am System überhaupt in der Lage sind, diese zu kontrollieren und damit die Verantwortung über den Systembetrieb zu übernehmen (z. B. Grote 2009). Denn es kann davon ausgegangen werden, dass die überwachenden Personen nicht in jedem Fall in der Lage sind, diesen Funktionen nachzugehen, da die funktionale und informationelle Distanz zum Systemablauf zu groß ist. Als beispielhaft sind hier Überwachungstätigkeiten anzusehen, die sich nicht direkt auf die physischen und stofflichen Anlagenprozesse auf dem Shop-floor beziehen, sondern etwa über Messwarten mediatisiert sind. Die Folge ist, dass ,the informal feedback associated with vibrations, sounds, and smells that many operaters relied upon“ eleminiert wird, daher das Bedienungspersonal die Anlagenzustände nicht mehr zutreffend einschätzen kann und unter Umständen falsche Entscheidungen in Hinblick auf Eingriffe in den automatischen Prozess trifft (Lee und Seppelt 2009). Daher ist vor allem auf die Grenzen der technischen Beherrschbarkeit der neuen Systeme auf Grund ihrer ausgeprägten Komplexität und ihrer inhärenten Unberechenbarkeiten zu verweisen. Die Automationsforschung spricht in diesem Zusammenhang von den ,ironies of automation“, wonach automatisierte Prozesse auf Grund ihres hohen Routinecharakters bei Störungen nur schwer zu bewältigende Arbeitssituationen erzeugen (Bainbridge 1983). In solchen Situationen seien Qualifikationen erforderlich, die im automatisierten Routinebetrieb nicht aufgebaut werden könnten (Windelband et al. 2011).

Arbeitssoziologischen Studien zu Folge sind dabei Handlungsweisen wie Intuition und Gespür, „Aus-dem-Bauch-heraus-Handeln“ oder auch Gefühl und Empathie gerade im Umgang mit komplexen Anlagen unverzichtbar - eine Seite von Arbeitshandeln, die als „subjektivierendes Arbeitshandeln“ gefasst werden kann (zusammenfassend Böhle 2013). Es geht dabei letztlich um ein Qualifikationsprofil, das durch eine Kombination von 
theoretischem Wissen und praktischer Erfahrung charakterisiert ist. Dieses spezifische Qualifikationsprofil ist als die zentrale Bedingung für eine kompetente Anlagenführung anzusehen, da es die Voraussetzung für ein improvisatorisch-experimentelles Arbeitshandeln im unvermeidbaren Störfall darstellt. Freilich muss durch entsprechende Systemgestaltung sichergestellt werden, dass die qualifizierten Arbeitskräfte auch in der Lage sind, ihren Überwachungsaufgaben effektiv nachzukommen (Schumann et al. 1994).

\section{Operative Arbeitsebene}

Eine weitere zentrale Dimension und Herausforderung ist die Gestaltung der Aufgaben und Tätigkeitsstrukturen auf der operativen Ebene im Kontext der smarten Produktionssysteme. Folgt man vorliegenden ersten Forschungsergebnisse, so lassen sich die absehbaren Entwicklungstendenzen wie folgt skizzieren (Ausschuss 2008; Kinkel et al. 2008; Windelband et al. 2011; Spath et al. 2013):

- Zum einen ist davon auszugehen, dass Arbeitsplätze mit niedrigen Qualifikationsanforderungen und einfachen, repetitiven Tätigkeiten durch intelligente Systeme in hohem Maße substituiert werden. Als Beispiele hierfür sind einfache Tätigkeiten in der Logistik, bei der Maschinenbedienung und bei der der bisher manuellen Datenerfassung und -eingabe zu nennen. In welchem Umfang Substitutionsprozesse aber eintreten werden, ist derzeit allerdings kaum abschätzbar.

- Zum Zweiten kann für die früher qualifizierte Facharbeiterebene eine Tendenz zur Dequalifizierung von Tätigkeiten befürchtet werden. $\mathrm{Zu}$ nennen sind hier Aufgaben wie einfachere Maschinenbedienung, material- und werkstoffbedingte Einstellungen sowie verschiedene Kontroll- und Überwachungsfunktionen, die automatisiert werden. Auch Dispositionsentscheidungen in der Produktionslogistik könnten mithilfe der neuen Systeme teilweise automatisiert werden. Denn benötigte Güter und Waren von Produktionsanlagen können weitgehend selbstständig angefordert werden, so dass die entsprechenden Steuerungsaufgaben der in der Fertigung eingesetzten Mitarbeiter entfallen. Sie greifen folglich nur noch in seltenen Ausnahmefällen in die Produktionsabläufe ein. In der Forschung wird daher von einer verbleibenden „Residualkategorie“ von qualifizierter Produktionsarbeit gesprochen, die jene Tätigkeiten umfasst, die nicht oder nur mit einem unverhältnismäßigen Aufwand automatisiert werden können. Dazu zählen etwa anspruchsvolle Wartungs- und Rüstaufgaben, bestimmte Einlegearbeiten, die Zuführung von Material und Halbfertigprodukten oder manuelle Produktionsfertigkeiten, die Experten- und Erfahrungswissen voraussetzen. Eine mögliche Konsequenz ist, dass die Betriebe nun niedriger qualifiziertes Personal als zuvor kostengünstig und ohne längere Anlernzeiten einsetzen können. Die Handlungsspielräume dieser Beschäftigtengruppe sind auf Grund strikter Systemvorgaben naturgemäß sehr eng.

- Zum Dritten kann aber auch eine Qualifikationsaufwertung und Tätigkeitsanreicherung erwartet werden. Als Grund hierfür können die erhöhte Komplexität der Fertigung und die informationstechnologischen Dezentralisierung von Entscheidungs-, Kontroll- und 
Koordinationsfunktionen angesehen werden. Daher werden die betroffenen Beschäftigten auf der operativen Ebene gefordert sein, zunehmend eigenständig zu planen und Abläufe abzustimmen. Erforderlich wird beispielsweise ein breiteres Verständnis über das Zusammenwirken des gesamten Produktionsprozesses, der Logistikanforderungen sowie der Lieferbedingungen. Neben dem steigenden Bedarf an Überblickswissen erlangen auch soziale Kompetenzen einen erhöhten Stellenwert, da mit der intensivierten Integration früher getrennter Funktionsbereiche der Bedarf an Interaktion - real wie computervermittelt - mit unterschiedlichen Personengruppen und weiteren Funktionsbereichen ansteigt. In der Forschung wird in diesem Zusammenhang das Schlagwort des „Facharbeiteringenieurs“ angeführt, mit dem zum Ausdruck gebracht werden soll, dass manuelle Fertigkeiten an Bedeutung verlieren, während zunehmend bestimmte Programmierkenntnisse sowie das Steuern, Führen und Einstellen von komplexen Systemen an Gewicht gewinnen.

Neben dem angesprochenen Aufgaben- und Qualifikationsanforderungen muss bei der Arbeitsgestaltung auf der operativen Arbeitsebene auch das mögliche hohe Kontrollpotential der neuen Systemtechniken in Rechnung gestellt werden. Die Fragen, welche Möglichkeiten sich hiermit verbinden und wie sie faktisch in Unternehmen genutzt werden, lässt sich derzeit kaum beantworten. In jedem Fall aber wird die Furcht vor dem durch die neuen technologischen Systeme möglichen „gläsernen Mitarbeiter“ ein wichtiger Einflussfaktor auf die Akzeptanz der neuen Technologien bei Beschäftigten und Arbeitnehmerinteressenvertretungen sein.

\section{Indirekte Bereiche und Leitungsebenen}

Fragt man, wie sich Produktionsarbeit in der hierarchischen Dimension verändert, so finden sich bislang nur wenig eindeutige Forschungsergebnisse. Höhere hierarchische Ebenen der Planungs- und Managementbereiche, so die Forschungsergebnisse, sind potentiell kaum direkt von einer Systemeinführung betroffen, jedoch dürfen sie bei der soziotechnischen Systemgestaltung keinesfalls vernachlässigt werden. Zusammenfassend kann man von widersprüchlichen „Ausstrahlungseffekten“ der Systemeinführung auf die hierarchische Ebene sprechen (Kinkel et al. 2008; Spath et al. 2013; Uhlmann et al. 2013):

- Zum einen deuten Evidenzen darauf hin, dass auf Grund der dezentralen Selbstorganisation der Systeme und einer entsprechend flexiblen Arbeitsorganisation auf der operativen Ebene ein Teil von bisher auf der Leitungsebene von technischen Experten und vom Produktionsmanagement ausgeführten Planungs- und Steuerungsfunktionen „nach unten“ abgegeben werden. Das heißt, mit Industrie 4.0-Systemen verbindet sich ein Dezentralisierungsschub und Hierarchieabbau innerhalb oft ohnehin schon relativ „flach“ strukturierter Fabrikorganisationen. 
- Zum Zweiten ist davon auszugehen, dass eine ganze Reihe von Aufgaben in indirekten Bereichen automatisiert und damit vereinfacht oder gar substituiert werden können. Je nach Systemauslegung kann es sich dabei Planungs- uns Steuerungsaufgaben, Tätigkeiten der Instandhaltung und des Service wie aber auch qualitätssichernde Aufgaben handeln.

- Zum Dritten dürften komplexitätsbedingt erweiterte und neue Planungsaufgaben auf diese Bereiche zukommen. Einige Hinweise deuten darauf hin, dass angesichts der Systemkomplexität Aufgaben des ,trouble shooting“ deutlich an Bedeutung gewinnen. Zudem kann davon ausgegangen werden, dass auf der Planungs- und Managementebene früher getrennte Aufgaben und Kompetenzen, beispielsweise IT- und Produktionskompetenzen, verschmelzen.

Verstärkt werden dürfte diese unklare Situation durch ein sich ebenso widersprüchlich wandelndes Kontrollpotential höher Positionsinhaber: Folgt man der Untersuchung von Kinkel et al. (2008), so eröffnen die Systeme und ihre informationstechnische Abbildung realer Prozessabläufe dem Produktionsmanagement neue und deutlich erweiterte Möglichkeiten zur Kontrolle der Prozesse und zur Störungsdiagnose. Es wird freilich auch auf das damit aufkommende Problem verwiesen, dass damit zugleich neuartige Probleme der Bewältigung und sinnvollen Filterung großer Datenmengen zu erwarten sein. Zugleich wird aber auch nicht ausgeschlossen, dass die Abläufe autonomer Systeme für die Planungsbereiche und die Produktionsleitungen auf Grund der ihrer Komplexität weitgehend intransparent bleiben und daher die bisherigen Entscheidungskompetenzen dieser Managementgruppe sich systembedingt auf die operative Ebene verlagern müssen. Als Konsequenz dieser Situation kann durchaus eine mangelnde Akzeptanz der neuen Technologien bei Managern befürchtet werden.

Obgleich sie bislang wenig eindeutig sind, lassen aber diese Hinweise den Schluss zu, dass die Planungs- und Managementbereiche in Folge der Einführung von Industrie 4.0Systemen längerfristig ebenso nachhaltig betroffen sein werden wir die operative Ebene. Mehr noch, es ist davon auszugehen, dass der Wandel und eine entsprechende Gestaltung auch der Leitungsebenen unverzichtbare Voraussetzung für die Beherrschung der neuen Technologien ist.

\section{Divergierende Muster der Arbeitsorganisation}

Resümiert man die vorliegenden Befunde über den Wandel von Tätigkeits- und Qualifikationsstrukturen, so wird zunächst deutlich, dass die Perspektive einer vollständigen Automatisierung und der menschenleeren Fabrik aus technologischen und ökonomischen Gründen keine realistische Perspektive darstellen kann (Ausschuss 2008). Zugleich ist aber auch kein „one-best-way“ der Entwicklung von Arbeit an smarten Produktionssystemen erkennbar. Auszugehen ist vielmehr von einem breiten Spektrum divergierender Muster der Arbeitsorganisation. Diese Muster bezeichnen alternative Möglichkeiten der Arbeits- 
Abb. 1 Polarisierte

Organisation, Quelle: Eigene

Darstellung

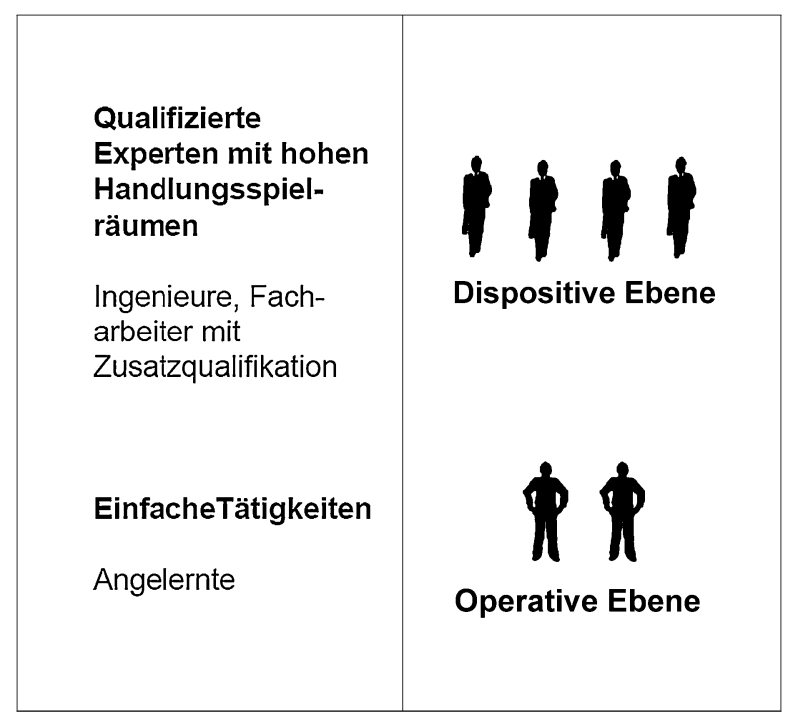

gestaltung an und in Industrie 4.0-Systemen. Das Spektrum der divergierenden Muster der Arbeitsorganisation wir durch zwei Pole begrenzt:

Der eine Pol entspricht einem Gestaltungsmuster, das auf den skizzierten Tendenzen der innerbetrieblichen Heterogenisierung von Aufgaben, Qualifikationen und Personaleinsatz beruht. Es finden sich in den Produktionssystemen einerseits eine vermutlich nur noch geringe Zahl einfacher Tätigkeiten mit geringem oder keinem Handlungsspielraum, die laufende standardisierte Überwachungs- und Kontrollaufgaben ausführen. Andererseits ist eine ausgeweitete oder auch neu entstandene Gruppe hoch qualifizierter Experten und technischer Spezialisten anzutreffen, deren Qualifikationsniveau deutlich über dem bisherigen Facharbeiterniveau liegt. Diesen Beschäftigten obliegen nicht nur dispositive Aufgaben etwa der Störungsbewältigung, sondern sie übernehmen verschiedentlich auch Aufgaben des Produktionsmanagements. Diese Beschäftigten sind, im Unterschied zu den Einfach-Beschäftigten, fraglos die Gewinner des absehbaren Technologieschubs. Dieses Muster der Arbeitsorganisation entspricht weitgehend den derzeit schon in vielen hoch technisierten Betrieben vorherrschenden Arbeitsformen, die als widersprüchliche Kombination von Gestaltungsprinzipien der Dezentralisierung und Aufgabenerweiterung einerseits und Strukturierung und Standardisierung andererseits gekennzeichnet werden kann (z. B. Kinkel et al. 2008; Hirsch-Kreinsen 2009; Abel et al. 2013). Insofern vermeiden die Betriebe neben den ohnehin aufwendigen technologischen Innovationen risikoreiche und mit Ungewissheit behaftete organisatorische Innovationen, wenn sie diesem etablierten Pfad arbeitsorganisatorischer Gestaltung folgen. Verkürzt soll daher dieses arbeitsorganisatorische Muster als Polarisierte Organisation bezeichnet werden (Abb. 1).

Der andere Pol des Spektrums wird von einem arbeitsorganisatorischen Muster gebildet, das metaphorisch als Schwarm-Organisation bezeichnet werden kann (Neef und Burmeister 2005; auch: Lee und Seppelt 2009; Cummings und Bruni 2009). Ziel dieser Orga- 
Abb. 2

Schwarm-Organisation, Quelle: Eigene Darstellung

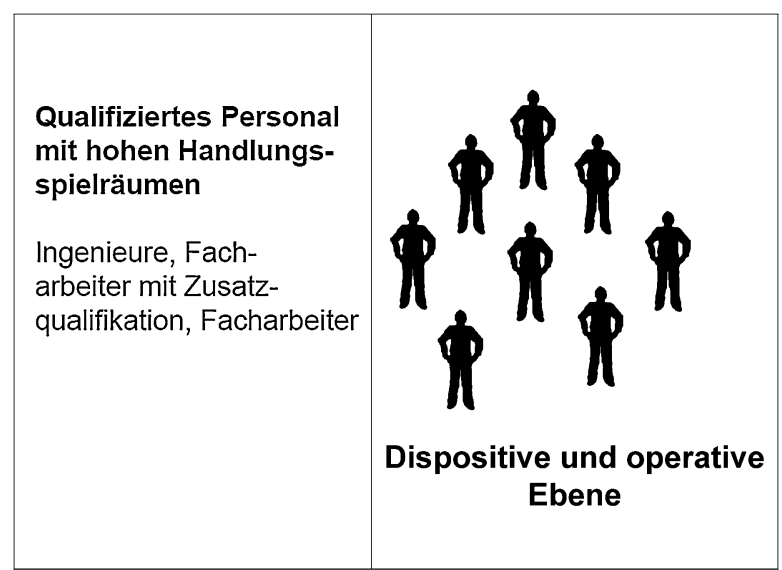

nisationsform ist es, durch höchstmögliche Offenheit und Flexibilität auf der Basis hoher Qualifikationen der Beschäftigten nicht antizipierbare Stör- und Sondersituationen jederzeit durch kompetentes und erfahrenes Arbeitshandeln bewältigen zu können. Diese Form der Arbeitsorganisation ist durch eine lockere Vernetzung sehr qualifizierter und gleichberechtigt agierender Beschäftigter gekennzeichnet. Einfache und niedrig qualifizierte Tätigkeiten sind hier nicht anzutreffen, denn sie sind weitgehend durch die Automatisierung substituiert worden. Zentrales Merkmal dieses Organisationsmusters ist, dass es keine definierten Aufgaben für einzelnen Beschäftigten gibt, vielmehr handelt das Arbeitskollektiv selbst organisiert, hoch flexibel und situationsbestimmt je nach zu lösenden Problemen im und am technologischen System. Allerdings existiert ein von der Leitungsebene vorgegebener Handlungsrahmen, der grundlegende Handlungsregeln, strategische Ziele und kollektive Orientierungen und Leitvorstellungen etwa in Hinblick auf einen möglichst störungsfreien und optimalen technologischen Prozess (Neef und Burmeister 2005). Anders formuliert, dieses Muster der Arbeitsorganisation zielt auf die explizite Nutzung informeller sozialer Prozesse der Kommunikation und Kooperation und der damit verbundenen extrafunktionalen Kompetenzen und des akkumulierten spezifischen Prozesswissens der Beschäftigten (Abb. 2).

\section{Stellhebel für die Arbeitsgestaltung}

Da es im Fall von smarten Produktionssystemen offensichtlich sehr unterschiedliche Gestaltungsmöglichkeiten von Produktionsarbeit gibt, liegt die Frage nahe, welche beeinflussbaren Stellhebel für die Gestaltung von Arbeit existieren. Fasst man die vorliegenden Literatur über die Einführung von komplexen Produktionssystemen zusammen, so spielen hierbei das jeweils von den Anwenderbetrieben verfolgte Automatisierungskonzept und damit zusammenhängend die Gestaltungs- und Einführungsprozesse der neuen Systeme eine grundlegende Rolle. 


\section{Alternative Automatisierungskonzepte}

Zwar ist grundsätzlich davon auszugehen, dass Automatisierungstechnologien die Gestalt der Arbeit keineswegs determinieren, jedoch können diese Spielräume je nach konkreter Systemauslegung sehr unterschiedlich sein. Fasst man die vorliegende Literatur zur Konzeption autonomer Produktionssysteme zusammen, so kann von divergierenden Systemkonzepten gesprochen werden (z. B. Hollnagel und Bye 2000; Kaber und Endsley 2004; Cummings und Bruni 2009; Lee und Seppelt 2009; Grote 2005):

- Zum einen kann von einem technologiezentrierten Automatisierungskonzept gesprochen werden. Diese Konzeption läuft auf eine weitreichende Substituierung von Arbeitsfunktionen durch die automatische Anlage hinaus. Die Rolle von menschlichem Arbeitshandeln hat in diesem Fall kompensatorischen Charakter. Ihm verbleiben Aufgaben, die nur schwer oder nicht zu automatisieren sind und sie umfassen generelle Überwachungsaufgaben. Anders formuliert, menschliches Arbeitshandeln hat in diesem Fall eine Lückenbüßerfunktion und der denkbare Endzustand einer solchen Systemauslegung ist die vollständige Automation. Es steht außer Frage, dass sich mit diesem Systemkonzept fortschreitend engere Spielräume für die Gestaltung von Arbeit verbinden.

- Zum anderen kann von einem komplementären Automatisierungskonzept gesprochen werden. Dieses Gestaltungskonzept richtet sich darauf, eine Aufgabenteilung zwischen Mensch und Maschine zu entwerfen, die eine zufriedenstellende Funktionsfähigkeit des Gesamtsystems ermöglicht. Dies setzt eine ganzheitliche bzw. kollaborative Perspektive auf die Mensch-Maschine-Interaktion voraus, die die spezifischen Stärken und Schwächen von menschlicher Arbeit und technischer Automatisierung identifiziert. Für die Gestaltung von Arbeit wird bei dieser Systemkonzeption ein technologischer Rahmen gesetzt, der in unterschiedlicher Weise genutzt werden kann.

In der einschlägigen sozialwissenschaftlichen Literatur (z. B. Grote 2005) wird übereinstimmend davon ausgegangen, dass allein eine komplementäre Systemauslegung eine hinreichende Voraussetzung für eine optimale Ausschöpfung der technologischen und ökonomischen Potentiale des automatisierten Produktionssystems darstellt. Denn sie überlässt nicht wie das technologiezentrierte Automatisierungskonzept menschlichem Arbeitshandeln lediglich fragmentierte Restfunktionen. Vielmehr eröffnet die komplementäre Konzeption Gestaltungsmöglichkeiten der Arbeit, die die oben genannten Awareness- und Feedback-Probleme des Handelns an komplexen Anlagen minimieren, informelles Arbeitshandeln und laufende Lernmöglichkeiten ermöglichen und damit eine hinreichende Kontrollierbarkeit des Systems möglich werden lassen. 


\section{Einführungsprozesse}

$\mathrm{Zu}$ betonen ist darüber hinaus, dass nicht nur der grundlegenden Entwicklungs- und Gestaltungsprozess der neuen Produktionssysteme, sondern auch der je konkrete Einführungsprozess der neuen Systeme bei Anwenderbetrieben eine entscheidende Rolle für die Gestaltung von Arbeit spielt. Denn erst im Verlauf der tatsächlichen Systemeinführung konkretisiert sich in der Regel die Gestaltung des gesamten sozio-technischen Systems auch in technischer, arbeitsorganisatorischer und personeller Hinsicht. Die Bedeutung des betrieblichen Einführungsprozesses für die letztendliche Systemauslegung und die sich durchsetzenden Muster von Produktionsarbeit begründet sich vor allem in dem Umstand, dass die neuen smarten Systeme in der Regel keineswegs schlüsselfertig in einem Plugand-Play-Verfahren in den Betrieben implementiert werden können. Denn es wird wohl nur selten der Fall eintreten, dass eine intelligente Fabrik als Gesamtkonzept auf die ,grüne Wiese“ gestellt wird. Vielmehr dürften die meisten autonomen Systeme zunächst einmal als Insellösungen innerhalb bestimmter Produktionssegmente in bestehende technischorganisatorischen Strukturen von Anwenderbetrieben integriert werden. Erforderlich wird daher im konkreten Einführungsfall ein unter Umständen langwieriger und aufwendiger wechselseitiger Abstimmungsprozess zwischen den neuen Systemen einerseits und den bestehenden betrieblichen Bedingungen andererseits. Verwiesen wird hier insbesondere auf den äußerst aufwendigen Abgleich der neuen Systeme mit vorhandenen Datenbeständen und Systemen (Spath et al. 2013; Schuh und Stich 2013). Insgesamt ist daher von lang laufenden Einführungs- und Anfahrphasen von Industrie 4.0-Systemen auszugehen, in deren Verlauf Tätigkeiten und Arbeitsorganisation eine hohe Flexibilität und Problemlösungsfähigkeit aufweisen müssen und dabei kaum einen definierbaren (End-)Zustand erreichen können.

Open Access This chapter is distributed under the terms of the Creative Commons Attribution Noncommercial License, which permits any noncommercial use, distribution, and reproduction in any medium, provided the original author(s) and source are credited.

\section{Literaturverzeichnis}

Abel, J., Ittermann, P., \& Steffen, M. (2013). Wandel von Industriearbeit. Herausforderung und Folgen neuer Produktionssysteme in der Industrie. Soziologisches Arbeitspapier Nr. 32, TU Dortmund.

Ausschuss für Bildung, Forschung und Technikfolgenabschätzung (2008). Zukunftsreport: Arbeiten in der Zukunft - Strukturen und Trends der Industriearbeit. Deutscher Bundestag Drucksache, 16, 7959.

Bainbridge, L. (1983). Ironies of automation. Automatica, 19(6), 775-779.

Böhle, F. (2013). Subjektivierendes Arbeitshandeln. In H. Hirsch-Kreinsen \& H. Minssen (Hrsg.), Lexikon der Arbeits- und Industriesoziologie, Berlin (S. 425-430).

Cummings, M., \& Bruni, S. (2009). Collaborative Human-Automation Decision Making. In S. Nof (Hrsg.), Handbook of automation, Berlin (S. 437-447). 
Forschungsunion/acatech (Hrsg.) (2013). Umsetzungsempfehlungen für das Zukunftsprojekt Industrie 4.0. Abschlussbericht des Arbeitskreises Industrie 4.0. Frankfurt am Main

Geisberger, E., \& Broy, M. (2012). Agenda CPS. Integrierte forschungsagenda cyber-physical systems. Heidelberg.

Grote, G. (2005). Menschliche Kontrolle über technische Systeme - Ein irreführendes Postulat. In K. Karrer, B. Gauss, \& C. Steffens (Hrsg.), Beiträge der Forschung zur Mensch-MaschineSystemtechnik aus Forschung und Praxis. Düsseldorf (S. 65-78).

Grote, G. (2009). Die Grenzen der Kontrollierbarkeit komplexer Systeme. In J. Weyer (Hrsg.), Management komplexer Systeme. München (S. 149-168).

Hirsch-Kreinsen, H. (2009). Innovative Arbeitsgestaltung im Maschinenbau? Soziologisches Arbeitspapier Nr. 27, TU Dortmund.

Hollnagel, E., \& Bye, A. (2000). Principles for modelling function allocation. International Journal of Human-Computer Studies, 52(2), 253-265.

Kaber, D., \& Endsley, M. (2004). The effects of level of automation and adaptive automation on human performance, situation awareness and workload in a dynamic control task. Theoretical Issues in Ergonomics Sciences, 5(2), 113-153.

Kinkel, S., Friedewald, M., Hüsing, B., Lay, G., \& Lindner, R. (2008). Arbeiten in der Zukunft: Strukturen und Trends der Industriearbeit. Berlin.

Kurz, C. (2013). Industrie 4.0 verändert die Arbeitswelt. In Gegenblende. Das gewerkschaftliche Debattenmagazin. www.gegenblende.de/24-2013 (15.01.2014).

Lee, J. D., \& Seppelt, B. (2009). Human factors in automation design. In S. Nof (Hrsg.), Handbook of automation, Berlin (S. 417-436).

Neef, A., \& Burmeister, K. (2005). Die Schwarm-Organisation - Ein neues Paradigma für das e-Unternehmen der Zukunft. In B. Kuhlin \& H. Thielmann (Hrsg.), Real-Time Enterprise in der Praxis. Berlin (S. 563-572).

Schuh, G. \& Stich, V. (Hrsg.) (2013). Produktion am Standort Deutschland. Ergebnisse der Untersuchung 2013. Aachen.

Schumann, M., Baethge-Kinsky, V., Kuhlmann, M., Kurz, C., \& Neumann, U. (1994). Trendreport Rationalisierung. Automobilindustrie, Werkzeugmaschinenbau, Chemische Industrie. Berlin.

Spath, D., Ganschar, O., Gerlach, S., Hämmerle, M., Krause, T., \& Schlund, S. (2013). Produktionsarbeit der Zukunft - Industrie 4.0. Stuttgart.

Sydow, J. (1985). Der soziotechnische Ansatz der Arbeits- und Organisationsgestaltung. Frankfurt am Main/New York.

Trist, E., \& Bamforth, K. (1951). Some social and psychological consequences of the long wall method of coal-getting. Human Relations, 4(1), 3-38.

Uhlmann, E., Hohwieler, E., \& Kraft, M. (2013). Selbstorganisierende Produktion mit verteilter Intelligenz. wt-online, 103(2), 114-117.

Windelband, L., et al. (2011). Zukünftige Qualifikationsanforderungen durch das „Internet der Dinge“ in der Logistik. In FreQueNz (Hrsg.) Zukünftige Qualifikationserfordernisse durch das Internet der Dinge in der Logistik, Zusammenfassung der Studienergebnisse, (S. 5-9). 